\title{
Schlafassoziierte Ventilationsstörungen - historische Entwicklung, Status quo und Ausblick
}

\section{Sleep-Related Breathing Disorders - Historical Development, Current Status, Future Prospects}

Autoren

Institute
H. Teschler ${ }^{1}$, W. Randerath ${ }^{2}$

Ruhrlandklinik, Abteilung Pneumologie, Westdeutsches Lungenzentrum, Universitätsklinikum Essen, Essen,

2 Institut für Pneumologie an der Universität Witten/Herdecke, Krankenhaus Bethanien, Solingen eingereicht 18.7.2010

akzeptiert nach Revision 21. 7.2010

\section{Bibliografie}

Dol http://dx.doi.org/

10.1055/s-0030-1255677

Pneumologie 2010; 64:

583-589 @ Georg Thieme

Verlag KG Stuttgart · New York

ISSN 0934-8387

Korrespondenzadresse Prof. Dr. med. Helmut Teschler

Ruhrlandklinik

Abteilung Pneumologie

Westdeutsches Lungenzentrum

Universitätsklinikum Essen

Tüschener Weg 40

45239 Essen

Helmut.Teschler@

Ruhrlandklinik.UK-Essen.de

\section{Zusammenfassung $\nabla$}

Schlafbezogene Atmungsstörungen zählen in den westlichen Ländern im Erwachsenenalter zu den Volkskrankheiten und werden klassifiziert als dominant obstruktive Schlafapnoe (OSA) oder als dominant zentrale Schlafapnoe (ZSA). Die Cheyne-Stokes-Atmung (CSA) ist Teil des Spektrums der ZSA. Die frühesten Beschreibungen von Patienten, die vermutlich an Schlafapnoe litten, finden sich in Fallbeispielen aus dem 19. Jahrhundert. Der Begriff „Pickwick-Syndrom“ für schläfrige Patienten mit Übergewicht und lautem Schnarchen wurde im Jahre 1889 eingeführt. Die ersten elektrophysiologischen Ableitungen des Schlafs von Pickwick-Patienten, bei denen außerdem die gestörte Atmung im Schlaf erkannt wurde, erfolgen in den späten 1950er- und 1960erJahren an den Universitäten zu Heidelberg und Freiburg. Der Begriff Schlafapnoe-Syndrom wurde von C. Guilleminault aus Stanford geprägt. Die Einführung der kontinuierlichen positiven Überdrucktherapie (engl.: continuous positive airway pressure, CPAP) durch C. E. Sullivan stellt einen enormen Impuls für die Weiterentwicklung der respiratorischen Schlafmedizin dar. Die zunehmende Anerkennung des Krankheitsbildes als weit verbreitetes Gesundheitsproblem wurde durch die Wisconsin-Studie unterstützt, die eine sehr hohe Prävalenz der Schlafapnoe in der Allgemeinbevölkerung der 30 bis 60 Jahre alten Bürger dieses amerikanischen Bundesstaates demonstrierte. Heutzutage gilt die obstruktive Schlafapnoe (OSA) als unabhängiger Risikofaktor für eine Reihe von klinischen Erkrankungen wie Arteriosklerose, Bluthochdruck, Herzinsuffizienz, Herzrhythmusstörungen, Schlaganfall, Diabetes mellitus und Depression. Dieser Artikel konzentriert sich auf Fragen und Probleme im Zusammenhang mit der OSA und der ZSA/CSA und befasst sich mit

\section{Abstract \\ $\nabla$}

Sleep-related breathing disorders are common adult illnesses in Western countries and classified as either dominant obstructive sleep apnoea or dominant central sleep apnoea. Cheyne-Stokes Respiration is part of the spectrum of CSA. The earliest descriptions of patients who presumably suffered from sleep apnoea were made in the 19th century. The term "Pickwickian" in connection with sleepy patients was introduced in 1889. The first electrophysiological sleep recordings of Pickwickian patients and the understanding of the syndrome as disordered breathing in sleep, were made during the late 1950s and 1960s at the universities of Heidelberg and Freiburg in Germany. The term sleep apnoea syndrome was introduced by Guilleminault from Stanford. The introduction of continuous positive airway pressure (CPAP) therapy by C. E. Sullivan and co-workers gave an enormous impetus to the field of sleep-disordered breathing. Its recognition as a public health problem was facilitated by the Wisconsin study, investigating the prevalence of sleep apnoea in the middle-aged general population. Nowadays obstructive sleep apnoea (OSA) is recognised as an independent risk factor for a wide range of clinical conditions, such as atherosclerosis, hypertension, heart failure, arrhythmias, stroke, diabetes, and depression. This article focuses on issues related to OSA and CSA/CSR, their pathogenesis, interaction with other comorbidities including cardiovascular diseases. Future research will focus on treatment effects on cardiovascular and metabolic outcomes in sleep apnoea and on the pathophysiological mechanisms responsible for the inflammatory state and cardiovascular morbidity in the syndrome. Other potential areas of research include biochemical markers, new diagnostic and therapeutic modalities. 
aktuellen Erkenntnissen zur Pathogenese und Interaktion von SBAS mit anderen Begleiterkrankungen wie beispielsweise den Herz-Kreislauf-Erkrankungen. Wichtige zukünftige Forschungsfelder betreffen die Untersuchung der Effekte etablierter und neuer Behandlungsmethoden auf kardiovaskuläre sowie metabolische Störungen infolge der Schlafapnoe und untersuchen die pathophysiologischen Mechanismen, die dem regionalen Entzündungsprozess zugrunde liegen, der zur kardiovaskulären Morbidität durch Schlafapnoe beiträgt. Andere mögliche Bereiche der Forschung umfassen biochemische Marker sowie neue diagnostische und therapeutische Modalitäten.

\section{Historischer Rückblick}

$\nabla$

Die Symptome und Charakteristika der obstruktiven Schlafapnoe - exzessive Tagesschläfrigkeit, lautes Schnarchen, unruhiger und nicht erholsamer Schlaf - sind so eindrucksvoll, dass man bei retrospektiver Betrachtung darüber verwundert sein könnte, dass die detaillierte Beschreibung und systematische Erforschung des Krankheitsbildes erst in den 70er-Jahren des vergangenen Jahrhunderts begann.

Der erste Fallbericht über einen Patienten mit Schlafapnoe stammt wahrscheinlich von Richard Caton aus Liverpool, der im Jahre 1889 zwar einen Patienten mit Übergewicht, Schnarchen und Einschlafattacken beschrieb, als wahrscheinlichste Ursache jedoch eine „Narkolepsie“ diskutierte. Im gleichen Jahr beschrieb A. Morison das klinische Bild eines Diamanthändlers, der übergewichtig war, schnarchte und an Tagesschläfrigkeit bzw. Einschlafattacken litt. Seinerzeit wies Christopher Heath auf die große Ähnlichkeit zwischen dem von Caton beschriebenen Fall mit Joe hin, der schläfrigen Person in Dickens Buch „The Posthumous paper of the Pickwick club“ aus dem Jahre 1837. Obwohl auch H. O. Sieker im Jahre 1955 einen Patienten mit den charakteristischen Zeichen und Befunden einer schweren Schlafapnoe darstellte, zitieren die meisten Autoren heute immer noch die Arbeit von C. S. Burwell aus dem Jahre 1956 als Erstbeschreibung eines Patienten mit Schlafapnoe-Syndrom.

Bahnbrechende Entwicklungen in der Physiologie und Neurophysiologie beflügelten die systematische Erforschung dieses Krankheitsbildes. Zu nennen sind die Entwicklung von Methoden für die Ableitung der Hirnströme (EEG), der Muskelpotenziale (EMG), der Augenpotenziale (EOG), für die kontinuierliche Registrierung der Atmung sowie der Sauerstoffsättigung $\left(\mathrm{SaO}_{2}\right)$. Das erste Elektroenzephalogramm am Menschen zeichnete der Psychiater H. Berger 1924 an der Universität in Jena auf. Im Jahre 1937 publizierte A. L. Loomis erste Daten über die Änderung der Hirnstromkurve im Schlaf. Bis zur Beschreibung des charakteristischen Schlafprofils mit Wechsel von Non-REM und REM-Schlaf sollten noch 15 Jahre vergehen. Das Team um den Schlafforscher N. Kleitmann in Chicago entdeckte erst 1953 die REM-Phasen. Die erste systematische Aufzeichnung eines Schlaf-EEGs (durchgeführt am Tag! ) bei einem Pickwick-Patienten wird W. Gerardy zugeschrieben, der diese Untersuchung im Jahre 1959 an der Universität Heidelberg durchführte und im Folgejahr publizierte. Bei Pickwick-Patienten beschrieb er nicht nur charakteristische Veränderungen in den Hirnstromkurven, sondern er erkannte auch den Zusammenhang zwischen diesem Syndrom und dem periodischen oder episodischen Auftreten von Apnoen. Des Weiteren berichtete er über eine deutliche Besserung von Schlafqualität und Tagesbefindlichkeit nach Gewichtsreduktion. Ein Jahr später wurden diese Erkenntnisse von D. B. Drachman und R. J. Gumnit am NIH in den USA bestätigt. Darüber hinaus wurde zum ersten Mal beschrieben, dass repetitive Atempausen im Schlaf bei diesen Patienten mit einer Desoxygenierung vergesellschaftet sind. Die Autoren vermuteten, dass die exzessive Schläfrigkeit von Patienten mit Pickwick-Syndrom durch eine $\mathrm{CO}_{2}$ „Vergiftung“ hervorgerufen wird. Die ersten Erkenntnisse von Schlaf-EEGs, die bei Pickwick-Patienten nicht am Tage, sondern tatsächlich in der Nacht aufgezeichnet wurden, gehen auf die Freiburger Neurologen Kuhlo und R. Jung zurück. Die Ergebnisse der 1960 begonnenen Registrierungen wurden 1964 auf einer internationalen Konferenz von Schlafforschern in Oberstdorf präsentiert, an der auch der Franzose H. Gastaut aus Marseilles und der Italiener Lugaresi aus Bologna teilnahmen. Von den Forschungsergebnissen von Kuhlo und Jung angeregt, begannen diese beiden Wissenschaftler mit eigenen Untersuchungen. Gastaut ergänzte die neurophysiologischen Untersuchungen als erster um Atmungsanalysen mit Hilfe von Mund- und Nasenkanülen und fand heraus, dass den Atempausen im Schlaf eine Okklusion der oberen Atemwege zugrunde liegt, wobei die Atemanstrengung in dieser Phase erhalten bleibt. Die Forschergruppe aus Bologna bestätigte ihrerseits die Beobachtungen von H. Gastaut und beschrieb erstmals drei Formen von Apnoen, die sie als obstruktiv, gemischt und zentral bezeichneten. Hypopnoen wurden dieser Terminologie kurz darauf von D. Kurtz aus Strasbourg hinzugefügt und in Verbindung gebracht mit Weckreaktionen und leichter Desaturation. Lugaresi führte gemeinsam mit P. V. Coccagna bei Pickwick-Patienten Kreislaufaufzeichnungen durch und erkannte als Erster die zum Teil dramatischen Blutdruckschwankungen, die mit den obstruktiven Apnoen im Schlaf im Zusammenhang stehen.

Basierend auf diesen neuen Erkenntnissen erfolgte 1969 an der HNO-Klinik in Freiburg die erste operative Behandlung der Schlafapnoe. Kuhlo veranlasste bei einem Patienten mit schwerstgradigem Pickwick-Syndrom und leichtem Koma die Durchführung einer Tracheotomie. Ziel dieser Operation war es, quasi einen Bypass für die im Schlaf okkludierten oberen Atemwege anzulegen. Bereits nach wenigen Tagen hatte sich der $\mathrm{Zu}-$ stand des Patienten erstaunlich gebessert. Er war tagsüber wach und litt nicht mehr unter Einschlafattacken. Die Arbeitsgruppe um Lugaresi bestätigte kurze Zeit später den Erfolg der Tracheotomie bei 6 eigenen Patienten mit schwerster Schlafapnoe und publizierte 1972 erstmals nicht nur die Normalisierung der EEGs, sondern auch eine Reduktion des zuvor erhöhten systolischen und diastolischen Blutdrucks dieser Patienten. Eine klinische Unterteilung des Pickwick-Syndroms in 10 Entitäten wurde 1972 von D. Caroll aus Baltimore vorgeschlagen. Nach intensiver Diskussion wurde diese komplexe Einteilung reduziert auf zwei Krankheitsbilder: nämlich das Pickwick-Syndrom mit und ohne Tagesschläfrigkeit.

Die nächsten systematischen Schritte in der Erforschung der Schlafapnoe wurden in den USA vorbereitet. Christian Guilleminault gelang 1973 der Nachweis, dass eine „Insomnie“ im Zusammenhang mit der Okklusion der oberen Atemwege im Schlaf auch bei normalgewichtigen Menschen vorkommt. Er berichtete 1977 außerdem darüber, dass viele Patienten mit Schlafstörungen der renommierten Schlafklinik in Stanford an einer schlafbezogenen Atmungsstörung erkrankt waren. Für dieses Krankheitsbild prägten die Autoren erstmals den Begriff „Schlafapnoe-Syndrom“. Einen der größten Meilensteine in der noch jungen Geschichte schlafassoziierter Ventilationsstörungen stellt ohne Zweifel die Entwicklung und Erstbeschreibung der nasalen kontinuierlichen Überdrucktherapie (engl.: nasal continuous positive airway pressure, nCPAP) im Jahre 1981 durch C. E. Sullivan und 


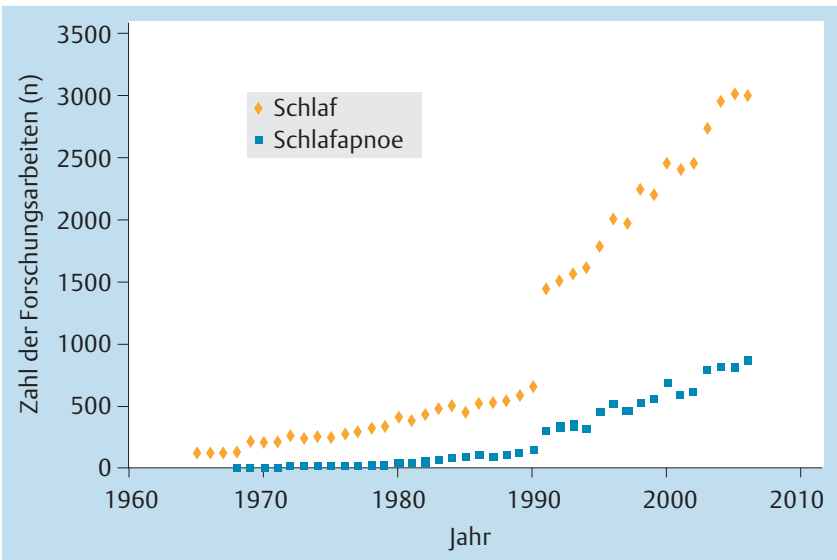

Abb. 1 Jährliche Anzahl der wissenschaftlichen Publikationen mit Schwerpunkt Schlaf und getrennt davon speziell fokussiert auf Schlafapnoe für den Zeitraum 1965 bis 2006 (Analyse des „ISI Web of Science; modifiziert nach [2])

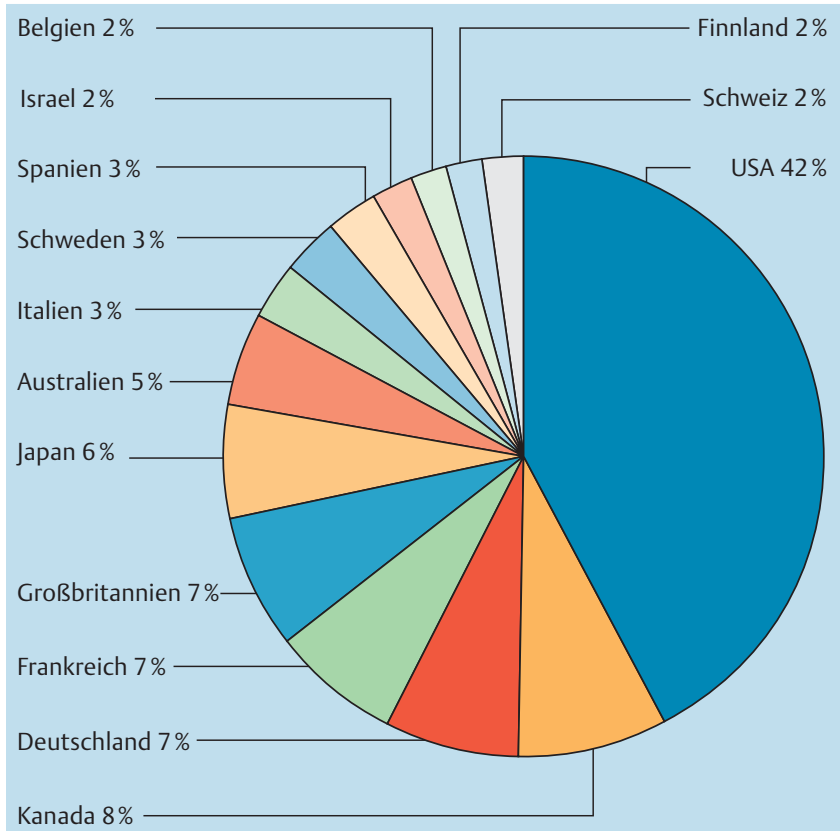

Abb. 2 Prozentuale Verteilung der publizierten Forschungsbeiträge, die von Wissenschaftlern aus verschiedenen Ländern zwischen 1965 und 2006 veröffentlicht wurde (Analyse des „ISI Web of Science; modifiziert nach [2]).

M. Berthon-Jones dar. Diese technische Innovation wurde kürzlich von der American Thoracic Society (ATS) als eine der 10 bedeutsamsten Entwicklungen in der Pneumologie im vergangenen Jahrhundert geehrt [1].

Um die Dynamik der schlafmedizinischen Forschungsaktivitäten - und damit die treibenden Kräfte in der Weiterentwicklung des Faches - im Hinblick auf den Beitrag einzelner Länder bzw. Kontinente und die am häufigsten zitierten Autoren und Originalarbeiten objektiv analysieren zu können, hat P. Lavie im Jahre 2007 die biometrische Datenbank „ISI Web of Science“ für den Zeitraum 1965 bis 2006 mit Hilfe der integrierten Suchmaschine unter Verwendung von vorab definierten Suchbegriffen ausgewertet [2]. Die Zahl der wissenschaftlichen Publikationen mit dem Schwerpunkt Schlafmedizin oder Schlafapnoe hat seit 1990 kontinuierlich zugenommen ( $\bullet$ Abb. 1).
Tab. 1 Die 20 produktivsten Autoren im Forschungsfeld „Schlafapnoe“ in der Zeit von 1965 bis 2006. Die Analyse bezieht sich auf die Auswertung im „ISI Web of Science" (modifiziert nach P Lavie [2]).

\begin{tabular}{|c|l|l|}
\hline Rang & Autor & Zahl der Publikationen \\
\hline 1 & Guilleminault C & 222 \\
\hline 2 & Peter JH & 102 \\
\hline 3 & Douglas NJ & 102 \\
\hline 4 & Redline S & 85 \\
\hline 5 & Smith DP & 80 \\
\hline 6 & Gozal D & 79 \\
\hline 7 & White DP & 79 \\
\hline 8 & Sullivan CE & 78 \\
\hline 9 & Series F & 76 \\
\hline 10 & Bradley TD & 73 \\
\hline 11 & Schwartz AR & 71 \\
\hline 12 & Hoffstein V & 69 \\
\hline 13 & Krieger J & 69 \\
\hline 14 & Levy P & 66 \\
\hline 15 & Pepin JL & 64 \\
\hline 16 & Stradling JR & 62 \\
\hline 17 & Lavie P & 60 \\
\hline 18 & Penzel T & 53 \\
\hline 19 & Hedner J & 52 \\
\hline 20 & Pack Al & 51 \\
\hline
\end{tabular}

Im Jahr 2006 entfielen immerhin ein Drittel aller schlafmedizinischen Originalarbeiten auf das Thema „Schlafbezogene Atmungsstörungen“. Die meisten Publikationen zum Themenkomplex Schlafapnoe bzw. schlafbezogene Atmungsstörungen wurden von Forschergruppen aus den USA beigesteuert, immerhin 36\% aus Europa, außerdem 5\% aus Australien, 6\% aus Japan und 8\% aus Kanada $(\bullet$ Abb. 2).

Vor diesem Hintergrund ist eine Ranking-Reihenfolge der 20 produktivsten Autoren in Abhängigkeit von der Häufigkeit der Zitierungen der jeweiligen Arbeitsgruppe ( $\bullet$ Tab. 1) und die Ermittlung der zehn am häufigsten zitierten Einzelpublikationen mit schlafmedizinischen Themen von Interesse ( $\bullet$ Tab. 2).

Der mit großem Abstand produktivste Autor war in diesem Zeitraum C. Guilleminault aus Stanford ( Tab.2). Aus deutscher Sicht erfreut es, dass J. H. Peter den zweiten Platz belegt und unter Berücksichtigung von T. Penzel auf Rang 18 immerhin zwei Autoren aus unserem Land in dieser Spitzengruppe zu finden sind. Doch nicht nur die Zahl der Publikationen ist bei dieser Art der Analyse der schlafmedizinischen Neuerungen und Meilensteine von zentraler Bedeutung, sondern auch die Exzellenz der einzelnen Arbeiten. Diesen Zusammenhang veranschaulicht die Gegenüberstellung der $\bullet$ Tab. 1 und 2. Die am meisten zitierte Arbeit auf dem Gebiet der Schlafapnoe war Ende 2006 die Publikation von T. Young zur Häufigkeit schlafbezogener Atmungsstörungen bei Erwachsenen im mittleren Lebensalter. In der Liste der Autoren mit den meisten Publikationen zum Themenkomplex ist T. Young aber nicht zu finden. Ähnliches gilt für Professor C. E. Sullivan, der mit der Erstbeschreibung der Behandlung der obstruktiven Schlafapnoe mit Hilfe der nasalen kontinuierlichen Überdrucktherapie (nCPAP) nicht nur eine der am häufigsten zitierten Publikationen in diesem Forschungsfeld publizierte, sondern der Schlafapnoeforschung damit nicht zuletzt aufgrund der kommerziellen und ökonomischen Aspekte in den Folgejahren die entscheidenden Impulse gegeben hat. Es dauerte nur wenige Jahre, bis die Behandlung der obstruktiven Schlafapnoe mit CPAP zum Goldstandard für alle Schweregrade der Erkrankung wurde, an deren Effizienz sich heutzutage alle anderen Behandlungs- 
Tab. 2 Die 10 am häufigsten zitierten Publikationen zum Thema „Schlafapnoe“ auf der Basis der Auswertung des „ISI Web of Science“ für den Zeitraum 1965 bis 2006 (modifiziert nach P Lavie [2]).

\begin{tabular}{|c|c|c|c|c|}
\hline Rang & Autoren & Titel & Journal & Anzahl der Zitate \\
\hline 1 & $\begin{array}{l}\text { Young T, Palta M, Dempsey J } \\
\text { et al. }\end{array}$ & $\begin{array}{l}\text { The occurrence of sleep-disordered } \\
\text { breathing among middle-age adults }\end{array}$ & $\begin{array}{l}\text { New England Journal of Medicine } \\
\text { 1993; 328: } 1230-1235\end{array}$ & 1946 \\
\hline 2 & $\begin{array}{l}\text { Remmers JE, Degroot WJ, } \\
\text { Sauerland EK et al. }\end{array}$ & $\begin{array}{l}\text { Pathogenesis of upper airway occlusion } \\
\text { during sleep }\end{array}$ & $\begin{array}{l}\text { Journal of Applied Physiology } \\
\text { 1978; 44: } 931-938\end{array}$ & 1088 \\
\hline 3 & $\begin{array}{l}\text { Sullivan CE, Berthon-Jones M, } \\
\text { Issa FG et al. }\end{array}$ & $\begin{array}{l}\text { Reversal of obstructive sleep-apnoea } \\
\text { by continuous positive airway pressure } \\
\text { applied through the nares }\end{array}$ & Lancet $1981 ; 1: 862-865$ & 954 \\
\hline 4 & $\begin{array}{l}\text { He J, Kryger MH, Zorick FJ } \\
\text { et al. }\end{array}$ & $\begin{array}{l}\text { Mortality and apnoea index in obstructive } \\
\text { sleep-apnoea - experience in 385-male } \\
\text { patients }\end{array}$ & Chest 1988; 94: 9-14 & 747 \\
\hline 5 & $\begin{array}{l}\text { Peppard PE, Young T, } \\
\text { Palta M et al. }\end{array}$ & $\begin{array}{l}\text { Prospective study of the association } \\
\text { between sleep-disordered breathing and } \\
\text { hypertension }\end{array}$ & $\begin{array}{l}\text { New England Journal of Medicine } \\
\text { 2000; } 342: 1378-1384\end{array}$ & 569 \\
\hline 6 & $\begin{array}{l}\text { Block AJ, Boysen PG, } \\
\text { Wynne JW et al. }\end{array}$ & $\begin{array}{l}\text { Sleep apnoea, hypopnea and oxygen } \\
\text { desaturation in normal subjects - strong } \\
\text { male predominance }\end{array}$ & $\begin{array}{l}\text { New England Journal of Medicine } \\
\text { 1979; 300: } 513-517\end{array}$ & 533 \\
\hline 7 & $\begin{array}{l}\text { Fujita S, Conway W, Zorick F } \\
\text { et al. }\end{array}$ & $\begin{array}{l}\text { Surgical-correction of anatomic abnorma- } \\
\text { lities in obstructive sleep-apnoea } \\
\text { syndromeuvulopalatopharyngoplasty }\end{array}$ & $\begin{array}{l}\text { Otolaryngology-Head and Neck } \\
\text { Surgery 1981; 89: } 923-934\end{array}$ & 507 \\
\hline 8 & $\begin{array}{l}\text { Nieto FJ, Young TB, Lind BK } \\
\text { et al. }\end{array}$ & $\begin{array}{l}\text { Association of sleep-disordered breath- } \\
\text { ing, sleep apnoea, and hypertension in } \\
\text { a large community-based study }\end{array}$ & $\begin{array}{l}\text { Journal of the American Medical } \\
\text { Association 2000;283: 1829-1836 }\end{array}$ & 472 \\
\hline 9 & $\begin{array}{l}\text { Guilleminault C, Stoohs R, } \\
\text { Clerk A et al. }\end{array}$ & $\begin{array}{l}\text { A cause of excessive daytime sleepiness - } \\
\text { the upper airway - resistance syndrome }\end{array}$ & Chest 1993; 104: $781-787$ & 446 \\
\hline 10 & $\begin{array}{l}\text { Hung J, Whitford EG, } \\
\text { Parsons RW et al. }\end{array}$ & $\begin{array}{l}\text { Association of sleep-apnoea with myo- } \\
\text { cardial-infarction in men }\end{array}$ & Lancet 1990; 336: $261-264$ & 407 \\
\hline
\end{tabular}

modalitäten messen lassen müssen. Deshalb verwundert es nicht, dass operative Behandlungsmethoden seither eine immer geringere Rolle spielen. Dies kommt auch in der aktuellen deutschen S3-Leitlinie „Nicht-erholsamer Schlaf“ zum Ausdruck, in der es heißt: „Operative Therapieformen können nur dann als alternative Verfahren erwogen werden, wenn konservative Maßnahmen keinen klinisch nachweisbaren Erfolg bringen oder nicht akzeptiert bzw. toleriert werden“ [3]. Wesentliche Impulse für die Weiterentwicklung der Schlafmedizin hat auch die Arbeitsgruppe von D. T. Bradley aus Toronto beigesteuert. Seine Arbeitsgruppe hat die Interaktion zwischen Herz, respiratorischer Kontrolle und Schlaf bei Patienten mit kongestiver Herzinsuffizienz systematisch untersucht und mit Evidenz-basierten Forschungsansätzen maßgeblich dazu beigetragen, dass dieses Feld aktuell im Fokus der interdisziplinären Schlafmedizin steht.

\section{Aktuelle Standortbestimmung}

Epidemiologische Studien im Bereich der Schlafmedizin haben unser Wissen über das Vorkommen schlafbezogener Ventilationsstörungen und die Auswirkungen selbiger auf Morbidität und Mortalität erweitert. Die Prävalenz von SBAS in der Allgemeinbevölkerung ist sehr hoch - es handelt sich um eine der häufigsten Volkskrankheiten bei Erwachsenen. In der oft zitierten Wisconsin-Studie wurde eine Stichprobe aus der arbeitenden Bevölkerung im Alter von 30 bis 60 Jahren untersucht ( $\bullet$ Tab. 2). Wird eine relevante SBAS anhand des Apnoe-Hypopnoe-Index (AHI) mit 5, 10 oder 15 respiratorische Ereignissen pro Stunde Schlaf definiert, litten in dieser Kohorte 24\%, 15\% bzw. 9\% der Männer und 9\%, 5\% bzw. 4\% der Frauen an einer Schlafapnoe. Der Anteil der Probanden mit Tagesschläfrigkeit und einem AHI $>5 / \mathrm{h}$ betrug in dieser Kohorte $4 \%$ bei Männern und $2 \%$ bei
Frauen. Für Deutschland wird angenommen, dass ungefähr $1 \%$ der Gesamtbevölkerung an einer behandlungsbedürftigen Schlafapnoe leidet. Eine vielfach höhere Prävalenz von SBAS bestätigte sich in Kohorten mit Herzinsuffizienz (HI), therapierefraktärer Hypertonie und anderen kardiovaskulären Erkrankungen [4,5]. Gegenüber der Allgemeinbevölkerung ist in diesen Stichproben der Anteil von Probanden mit ZSA mit oder ohne CSA überproportional stark erhöht. Eine SBAS fand sich bei $76 \%$ von 700 Patienten einer Kohorte mit HI. Davon litten 40\% an einer dominant zentralen und 36\% an einer dominant obstruktiven Schlafapnoe. Die Patienten mit ZSA/CSA in der Studie von Oldenburg und Mitarbeitern waren charakterisiert durch eine geringere linksventrikuläre Auswurffraktion (LVEF) und eine höhere NYHA-Klasse als in der Gruppe mit OSA (zit. in 3). Mehrere epidemiologische Studien dokumentieren einen klaren Zusammenhang zwischen SBAS und kardiovaskulärer Morbidität und Mortalität. In der Sleep-Heart-Health-Studie (SHHS) hatten Probanden mit einem AHI > 10/h mehr kardiovaskuläre Ereignisse als Probanden ohne relevante Schlafapnoe [6]. Ein erhöhter AHI und ein erhöhter Hypoxämie-Index (Zeit mit $\mathrm{SaO}_{2}<90 \%$ ) waren assoziiert mit größerer linksventrikulärer Masse und verminderter linksventrikulärer Auswurffraktion. Probanden mit einem AHI von 30/h und mehr hatten häufiger Vorhofflimmern und ventrikuläre Tachykardien oder komplexe ventrikuläre Herzrhythmusstörungen als Probanden ohne SBAS.

Kürzlich wurden die Mortalitätsergebnisse der Wisconsin-SleepKohorte ( $n=1522$ Patienten) im zeitlichen Verlauf von 18 Jahren publiziert ( Tab.2). Anhand der Resultate der PSG zum Zeitpunkt der Rekrutierung der Kohorte wurden mit Hilfe des AHI 3 Gruppen gebildet. Unterschieden wurde zwischen einer leichten Schlafapnoe $(A H I \geq 5 / h)$, einer mittelschweren $(A H I \geq 15 / h)$ und einer schweren $(A H I \geq 30 / h)$ SBAS. Selbst bei Adjustierung der Risikoanalyse für Alter, Geschlecht, BMI und andere Ko-Fak- 


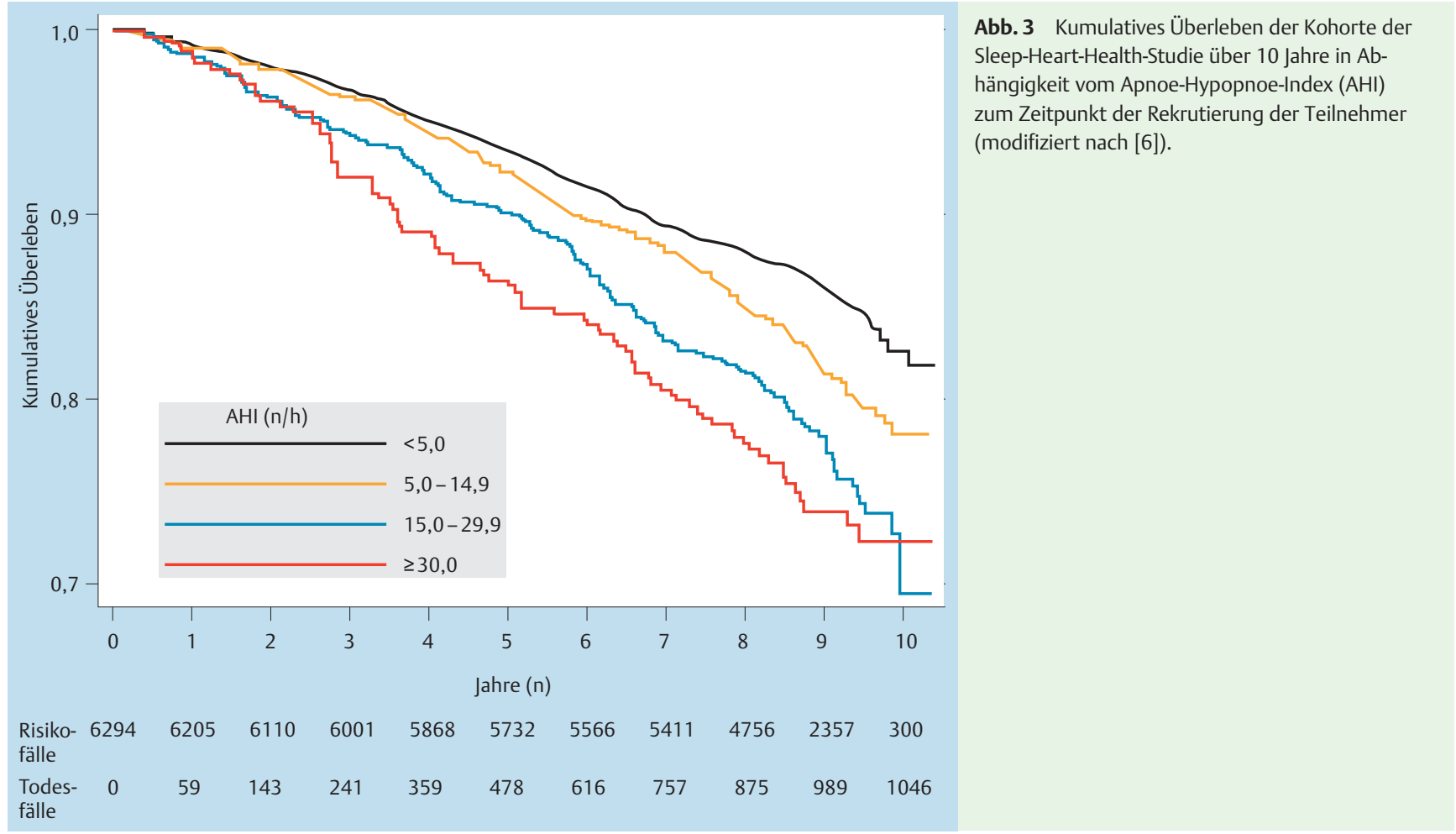

toren fand sich eine signifikante Zunahme der Gesamtmortalität mit dem Schweregrad der Schlafapnoe. Die adjustierte HazardRatio (HR) für die Gesamtmortalität betrug beim Vergleich der Gruppen mit schwerer und fehlender Schlafapnoe 3,0 und für die kardiovaskuläre Mortalität 5,2. Zwei weitere Studien, nämlich die Sleep-Heart-Health-Studie und die Busselton-Health-Studie, bestätigen den Zusammenhang zwischen dem Schweregrad der Schlafapnoe und dem Sterblichkeitsrisiko der rekrutierten Patienten $(\bullet$ Abb. 3) [6].

Neue Erkenntnisse über die Pathophysiologie der SBAS haben wesentlich zum besseren Verständnis der Variabilität und insbesondere der hohen Prävalenz der Schlafapnoe bei kardiovaskulären Erkrankungen (z.B. Herzinsuffizienz, HI) beigetragen. Erst seit kurzem ist die Bedeutung der Verlagerung von Flüssigkeit aus den Beinen in den Hals unmittelbar nach dem Hinlegen als genereller Mechanismus für die Entstehung einer Schlafapnoe bekannt, wobei das Ausmaß der Flüssigkeitsverlagerung in Beziehung steht zum Schweregrad und zum dominanten Typ der Schlafapnoe [5].

Bei Patienten mit koronarer Herzkrankheit (KHK) findet sich häufiger als bei Gesunden eine OSA. Und umgekehrt leiden Patienten mit OSA signifikant häufig an einer KHK oder allgemeinen Gefäßsklerose [5]. Es wird angenommen, dass die repetitiven Hypoxien und die Arousals im Zusammenhang mit Apnoen zu einer Erhöhung des sympathischen Tonus und einer systemischen Entzündung beitragen, die sich auf das Endothel der Gefäße negativ auswirkt. Deshalb verwundert es nicht, dass respiratorische Ereignisse im Schlaf eine messbare Störung der Gefäßregulation verursachen und den Umbau der Gefäßwände perpetuieren. Es resultiert eine gestörte blutflussvermittelte Gefäßdilatation und eine signifikante Zunahme der Intima-Media-Dicke. Dabei besteht eine signifikante Beziehung zwischen dem Ausmaß dieser Veränderungen und dem Schweregrad der SBAS.
Die Schlafapnoe erhöht das relative Risiko für eine arterielle Hypertonie unabhängig von anderen bekannten Ko-Faktoren [4-6]. In der Sleep-Heart-Health-Studie fand sich unter mehr als 6000 Probanden eine lineare Beziehung zwischen dem mittleren systolischen und diastolischen Blutdruck und dem Schweregrad der obstruktiven Schlafapnoe [6]. Außerdem zeigte sich eine lineare Zunahme der Prävalenz der arteriellen Hypertonie mit dem Schweregrad einer obstruktiven Schlafapnoe. Auch in der Wisconsin-Studie ( Tab.2) fand sich eine Dosis-Wirkungsbeziehung zwischen der Schwere der SBAS und der Manifestation einer Hypertonie. Bei einem AHI von 15/h und mehr Ereignissen zum Zeitpunkt der Rekrutierung betrug die OR für die Entwicklung einer Hypertonie in der Beobachtungszeit von 4 Jahren selbst nach Kompensation für alle bekannten Risikofaktoren 2,89. Die Behandlung der OSA mittels CPAP reduziert die Blutdruckschwankungen und darüber hinaus die sympathische Aktivität im Schlaf [4-6]. Der Effekt ist variabel und vom Schweregrad der Hypertonie, der Schlafapnoe und dem Ausmaß der Tagesschläfrigkeit abhängig.

Die Sleep-Heart-Health-Studie konnte ein höheres Risiko für die Entwicklung komplexer Herzrhythmusstörungen bei Patienten mit SBAS im Vergleich zu Kontrollen belegen [5,6]. So fand sich bei Probanden mit einem AHI > 30/h gegenüber Kontrollen ohne signifikante Schlafapnoe eine 4-fache Zunahme von Vorhofflimmern, eine 3,4-fache Zunahme von ventrikulären Tachykardien und eine 1,7-fache Zunahme von komplexen ventrikulären Ektopien [6].

Bei mehr als 50\% der Patienten mit HI findet sich eine SBAS, wobei häufiger eine OSA als eine ZSA vorliegt und polysomnografisch nicht selten eine Kombination beider Schlafapnoetypen oder eine überwiegende OSA mit periodischem, CSA-ähnlichem Atmungsmuster erkennbar ist. Von großer klinischer Relevanz ist die Erkenntnis, dass Patienten mit HI seltener an Tagesschläfrigkeit als charakteristischem Symptom einer Schlafapnoe leiden $[4-6]$. 
Bei Patienten mit kongestiver Herzinsuffizienz und ZSA/CSR verbessert CPAP zwar die SBAS, die nächtliche Oxygenierung und die Ejektionsfraktion, doch sprechen die Daten der CanPAP-Studie gegen eine Reduktion der Schlaffragmentierung und der Sterblichkeit durch Behandlung mit CPAP. Mit der adaptiven Servoventilation (ASV) steht seit 2001 eine intelligente PAP-Methode zur Verfügung, die eine erfolgreiche Therapie der ZSA/CSR und darüber hinaus die simultane Behandlung einer zusätzlich vorhandenen OSA ermöglicht [5]. Im Hinblick auf die Verbesserung der ZSA/CSR und der Schlafqualiät von Patienten mit HI ist die ASV der nasalen CPAP- und Sauerstofftherapie weit überlegen. Ob ASV das Überleben von Patienten mit HI und SABS verbessert, wird derzeit in klinischen Studien (Serve-HF, Advent-HF) geprüft, die frühestens 2014 abgeschlossen sein werden.

Die obstruktive Schlafapnoe ist ein (unabhängiger) Risikofaktor für die Entwicklung eines Schlaganfalls, die zentrale Schlafapnoe hingegen im Regelfall eine Konsequenz des Insults. Die Behandlung der Schlafapnoe mit CPAP reduziert zwar das Risiko für die Manifestation kardiovaskulärer Ereignisse und verbessert die Prognose nach einem zerebrovaskulären Ereignis, doch tolerieren nur wenige Schlaganfallpatienten diese Therapie.

Einen weiteren Brennpunkt bildet in den letzten Jahren die Erforschung des Zusammenhangs zwischen schlafbezogener Ventilationsstörung und Diabetes mellitus bzw. metabolischem Syndrom. Die obstruktive Schlafapnoe ist mit einer erhöhten Insulinresistenz verbunden, die mit dem Schweregrad der Schlafapnoe korreliert. Die Behandlung der Schlafapnoe wirkt sich positiv auf den Nüchtern-Blutzucker, die HbA1C-Spiegel und die Insulinsensitivität aus.

Großer Forschungsbedarf besteht nach wie vor bezüglich des Zusammenhangs zwischen dem Ausmaß der Tagesmüdigkeit/schläfrigkeit und den Effekten der Therapie. Zahlreiche Studien belegen unzweifelhaft, dass symptomatische Patienten mit Schlafapnoe die Behandlung mit CPAP besser tolerieren und der blutdrucksenkende Effekt von CPAP bei Hypertonikern mit OSA mit dem Ausmaß der Tagesschläfrigkeit korreliert. Außerdem scheint das Alter eine modulierende Rolle zu spielen, da mehrere Studien zeigen konnten, dass ältere Menschen mit Schlafapnoe weniger gefährdet sind als jüngere.

Für eine umfangreichere Standortbestimmung bezüglich der aktuellen Diagnostik und Therapie schlafassoziierter Ventilationsstörungen sei der interessierte Leser auf die neue S3-Leitlinie „Nicht-erholsamer Schlaf“ der Deutschen Gesellschaft für Schlafmedizin und Schlafforschung (DGSM) verwiesen [3].

\section{Zukunftsaspekte}

$\nabla$

Albert Einstein sagte: „Der Unterschied zwischen Vergangenheit, Gegenwart und Zukunft ist eine Illusion, wenn auch eine hartnäckige." Obwohl dem nichts hinzuzufügen ist, wird nachfolgend ein Blick in die nähere Zukunft gewagt.

Der DAK-Gesundheitsreport 2010 belegt, dass nicht erholsamer Schlaf eines der größten Gesundheitsprobleme in Deutschland darstellt [7]. Mehr als 50\% der Erwerbstätigen in Deutschland leiden unter Schlafstörungen. Neun Prozent der Betroffenen verspüren sehr oft den Drang, am Arbeitsplatz einzuschlafen. Dieses Risiko nimmt mit dem Schweregrad der Schlafstörung zu. Fast jeder Zehnte hat hochgradige Schlafprobleme - in ganz Deutschland entspricht dies zirka 3,5 Millionen Erwerbstätigen. Vor diesem Hintergrund besteht unverzüglicher Handlungsbedarf in
Klinik, Forschung und Lehre, aber auch ein Umdenken in der aktuellen Gesundheitspolitik.

Zukünftige Forschungsfelder ergeben sich aus den offenen Fragen zur Epidemiologie, Pathogenese, Diagnostik und Therapie SBAS. Sie betreffen außerdem die bidirektionale Interaktion zwischen der Schlafapnoe und anderen Erkrankungen, d.h. die Auswirkungen einer Schlafapnoe als Komorbidität auf andere Erkrankungen und andererseits die Effekte anderer Gesundheitsstörungen auf die Entstehung oder die Modulation einer bestehenden Schlafapnoe. Hier sind beispielhaft das Opiat-induzierte SchlafapnoeSyndrom oder die Interaktion von Apnoen/Hypopnoen und der respiratorischen Insuffizienz sowie die kardiovaskulären Reaktionen auf diese Ereignisse zu nennen. Vor diesem Hintergrund sollten sich zukünftige Forschungsanstrengungen unter anderem auf die Interaktion der Schlafapnoe mit Arteriosklerose, Hypertonie, Herzrhythmusstörungen, Diabetes mellitus und metabolischem Syndrom fokussieren. Ein hoher Stellenwert kommt zudem der Entwicklung und Validierung neuer Therapiekonzepte jenseits von CPAP oder ASV zu. Die Forschung muss Antworten auf die Frage nach Alternativen zur CPAP-Therapie (Operationen, Medikamente etc.) finden. Außerdem muss die Entwicklung zuverlässiger, aber dennoch preiswerterer und flächendeckend verfügbarer Methoden für die Diagnostik schlafbezogener Ventilationsstörungen vorangetrieben werden. Von großer Bedeutung erscheint zudem die Entwicklung von neuen Indizes für die Bemessung des Schweregrads einer Schlafapnoe sowie für die Klassifikation komplexer schlafassoziierter Ventilationsstörungen. Welche Rolle die Genetik spielt und ob biochemische Parameter zur Diagnostik, Schweregradbeurteilung und Risikostratifizierung SBAS herangezogen werden können, bedarf der systematischen Erforschung. Analog zu anderen chronischen Erkrankungen ist die Etablierung von Forschungsverbünden und von systematischer Netzwerkforschung eine überfällige Maßnahme zur Verbesserung der Infrastruktur für die forschende Schlafmedizin und die Generierung dringend benötigter Daten über die Epidemiologie, die erzielbaren Therapieeffekten und die sozioökonomischen Kosten von SBAS in unserem Land, sei es infolge von nicht erkannten oder von behandelten Patienten mit Schlafapnoe.

Der berühmte amerikanische Schlafforscher Bill Dement hat einmal gesagt: „Der Mensch ist nicht gesund, solange sein Schlaf nicht gesund ist“. Folglich sollte jeder Arzt über ausreichende schlafmedizinische Kenntnisse verfügen. Eine große Herausforderung besteht ohne Zweifel darin, die schlafmedizinische Lehre - und damit das schlafmedizinische Wissen von Ärzten und anderen in der Versorgung tätigen Akteuren im Gesundheitswesen - durch curriculare Weiterbildung zu verbessern.

Im scharfen Kontrast zur enormen Häufigkeit und gesundheitlichen Relevanz von Schlafstörungen im allgemeinen sowie von schlafbezogenen Atmungsstörungen im speziellen stehen die gesundheitspolitischen Entscheidungen zur Weiterentwicklung dieses noch recht jungen Bereichs der Medizin. Die Deutsche Forschungsgemeinschaft wendet nur einen winzigen Bruchteil ihrer Fördermittel für die schlafmedizinische Forschung auf, eigenständige schlafmedizinische Lehrstühle wurden an unseren Universitäten und sonstigen Forschungseinrichtungen bislang nicht etabliert, in der Versorgungsforschung spielt die Schlafmedizin nach wie vor kaum eine Rolle, obwohl kein Zweifel an einer eklatanten schlafmedizinischen Unter- und Fehlversorgung besteht. Einerseits ist die stationäre und ambulante Schlafmedizin sowohl bei Abrechnung auf DRG-Basis wie bei Vergütung über Regelleistungsvolumen (Entscheidung des Bewertungsausschusses vom 
26. 3.2010) erheblich unterfinanziert, andererseits resultiert daraus schon heute eine Stagnation - wenn nicht sogar eine Reduktion - der mit hohem Aufwand aufgebauten schlafmedizinischen Infrastruktur, Fachkompetenz und vor allen Dingen der vergleichsweise guten Qualität der schlafmedizinischen Versorgung in Deutschland. Die Unterteilung in eine ambulante und stationäre Schlafmedizin mit der Forderung nach ambulanter vor stationärer Versorgung ist ein nicht zu akzeptierendes Spezifikum des deutschen Gesundheitssystems. Dadurch werden keine echten Versorgungsprobleme gelöst, sondern gesundheitsökonomisch falsche Anreize und Hemmnisse aufgebaut. Es ist an der Zeit, diese Hürden zu überwinden und die zukünftige Ausrichtung der Schlafmedizin in Deutschland zum Gegenstand der Versorgungsforschung zu machen. Nur so lässt sich die Frage nach den bestmöglichen qualitätsgesicherten Strukturen und Prozessen beantworten, die zukunftsorientiert sind und nicht auf dem Rücken der Patienten und Ärzteschaft ausgetragen werden. Die Verkürzung der Schlafmedizin auf die Schlafapnoemedizin oder „Schnarchmedizin“ ist eine fatale Fehlentwicklung, die zurückzuführen ist auf die Zulassungsbeschränkungen für die ambulante Schlafmedizin und den nicht unerheblichen Einfluss der Medizingeräteindustrie in diesem Bereich. Es ist zu fordern, nach amerikanischem Vorbild auch in Deutschland Lehrstühle für Schlafmedizin zu gründen, die schlafmedizinische Grundlagenforschung mit öffentlichen Geldern (z.B. DFG) zu stärken und evidenzbasierte schlafmedizinische Versorgungsstrukturen aufund auszubauen. Es ist an der Zeit, dass die Gesundheitspolitik „aufwacht“ und die Voraussetzungen für eine angemessene schlafmedizinische Versorgung auf allen Ebenen des Gesundheitswesens (Hausarzt, Facharzt, Schlafspezialist) schafft. Und das ist gut investiertes Kapital. Denn Schlafmedizin ist ein Paradebeispiel für erfolgreiche Prävention mit großem Potenzial für eine vorausschauende Problemvermeidung in den Primär-, Sekundär- und Tertiärfeldern der Präventivmedizin.

\section{Interessenkonflikte}

Winfried Randerath: Der Autor ist als Principal Investigator oder Co-Investigator an mehreren Studien Therapie der SchlafapnoeSyndrome beteiligt. Er ist beratend tätig für die Firmen Weinmann und Linde und hat Honorare für Referate von den Firmen Weinmann, ResMed, Respironics sowie MSD erhalten.

Helmut Teschler: Der Autor ist Principal Investigator der internationalen Serve-HF Studie, die den Effekt von ASV bei Herzinsuffizienz untersucht. Er war Mitglied in verschiedenen Advisory Boards mit schlafmedizinischem Schwerpunkt (Cephalon, ResMed, Sanofi-Aventis) und hat für Referate mit schlafmedizinischem Schwerpunkt Honorare bezogen von den Firmen Boehringer-Ingelheim, Cephalon, ResMed und Sanofi-Aventis.

\section{Literatur}

1 Wallace J. Colleagues in discovery: one hundred years of improving respiratory health. SanDiego, CA: TehabiBooks, 2005

2 Lavie $P$. Who was the first to use the term Pickwickian in connection with sleepy patients? History of sleep apnoea syndrome. Sleep Medicine Reviews 2008; 12: 5-17

3 Deutsche Gesellschaft für Schlafforschung und Schlafmedizin (DGSM). S3-Leitlinie Nicht erholsamer Schlaf/Schlafstörungen. Somnologie 2009; 13: 4- 160

4 Eastwood J, Malhotra A, Palmer LJ et al. Obstructive Sleep Apnoea: From pathogenesis to treatment: Current controversies and future directions. Respirology 2010; 15: 587-595

5 Logan AG, Bradley TD. Sleep apnea and cardiovascular disease. Curr Hypertens Rep 2010; 12: $182-188$

6 Sleep heart health study. www.jhucct.com/shhs/; Stand: 28. 7. 2010

7 DAK Gesundheitsreport 2010. www.iges.de/publikationen/gutachten/ dak_report_2010/index_ger.html; Stand: 24.7.2010 